\title{
Effect of hypothermia on respiratory rhythm generation in hamster brainstem-spinal cord preparations
}

\author{
M. Beth Zimmer*, W.K. Milsom \\ University of British Columbia, Department of Zoology, 6270 University Blvd., Vancouver, BC, Canada V6R 1G8
}

Accepted 7 June 2004

\begin{abstract}
This study examined the effect of hypothermia on respiratory neural output from brainstem-spinal cord preparations of a cold tolerant rodent, the Syrian hamster. Brainstem-spinal cords from neonatal hamsters (0-6 days) were placed in a recording dish and respiratory-like neural activity was recorded from roots of the first cervical nerve. The preparations were cooled and warmed in a continuous or stepwise fashion. Inputs from the pons completely inhibited neural activity under steady state conditions. With the pons removed, fictive breathing was robust. Cooling caused respiratory arrest, followed by spontaneous resumption of activity on re-warming. Preparations from older hamsters (4-6 days old) were more cold tolerant than younger preparations ( $0-3$ days old). Motor discharge was episodic during continuous cooling, and seizure-like discharge was observed during continuous warming. These phenomena were not observed with stepwise temperature changes suggesting that transient temperature effects on membrane properties may be involved. These preparations were not as cold tolerant as hamster pups in vivo but they retained the ability to autoresuscitate at all ages studied.
\end{abstract}

(c) 2004 Elsevier B.V. All rights reserved.

Keywords: Hypothermia; Respiratory pattern generation; Mammals; Syrian hamster; Pattern of breathing; Respiratory rhythm generation; Hypothermia

\section{Introduction}

At birth all mammals are extremely cold tolerant but they gradually lose this tolerance as they mature and develop (Adolph, 1951). There is large species variation in both the extent of neonatal cold tolerance as well as in the difference in cold tolerance

\footnotetext{
* Corresponding author. Present address: Department of Anatomy and Cell Biology, School of Medicine, Wayne State University, 540 East Canfield, Detroit, MI 48201, USA.

Tel.: +1-313-577-8143; fax: +1-313-577-3125.

E-mail address: mzimmer@med.wayne.edu (M.B. Zimmer).
}

between neonates and adults. For example, neonatal rats survive $>30^{\circ} \mathrm{C}$ reductions in body temperature whereas adults can not survive much more than a $10^{\circ} \mathrm{C}$ reduction in body temperature. In a hibernating species such as the hamster both neonates and adults survive $>30^{\circ} \mathrm{C}$ reductions in temperature (Adolph, 1951). Neonatal hamsters are still more cold tolerant than adult hamsters, but the difference is only a couple of degrees. The reasons for the differences in the responses to hypothermia between non-hibernators (rats) and hibernators (hamsters), as well as between animals of different ages, are not well understood. 
Hibernating species have long been recognized for their extreme tolerance to the cold. Every winter, during entrance into hibernation, body temperature, metabolism, ventilation and heart rate are dramatically reduced. These variables are maintained at new steady, low levels throughout each hibernation bout (3-14 days), after which, animals arouse and these variables return to euthermic values. This cyclic behaviour of hibernation (entrance, steady state hibernation, arousal, and euthermia) continues throughout the entire hibernation season and while these processes are well described, the mechanisms underlying the physiological changes that accompany them are only partially understood (see Lyman, 1982, for review).

In addition to extreme cold tolerance, neonatal mammals have the impressive ability of being able to auto-resuscitate after respiratory and cardiac arrest due to hypothermia. Adolph (1948a) found that during severe hypothermia, ventilation and heart rate stopped in some neonatal rats and when the rats were re-warmed, ventilation and heart rate spontaneously restarted. Adult mammals, on the other hand, cannot auto-resuscitate. If ventilation stops during exposure to hypothermia, immediate ventilatory assistance is required to maintain respiration and life (rat, Adolph, 1948a,b). The respiratory-like motor output of neonatal rat brainstem-spinal cord preparations also stops in response to cold and restarts during re-warming much as it does in vivo (Mellen et al., 2002). In this case, the arrest can be attributed to a direct action of cold on the medullary rhythm generator and not on premotor or motor neurons (Mellen et al., 2002).

Given species differences in neonatal cold tolerance and the extent to which this cold tolerance is lost in adults, the present study was designed to evaluate the effect of hypothermia on the respiratory motor output of developing ( $0-6$ days old) brainstem-spinal cord preparations of a hibernating species, the Syrian hamster. Since the cold tolerance of neonatal hamsters in vivo is greater than the cold tolerance of neonatal rats (Adolph, 1951) and is not lost to the same extent in adults, we predicted that hamster brainstem-spinal cord preparations would be more cold tolerant than rat brainstem-spinal cord preparations and would also be able to autoresuscitate. Furthermore, episodic patterns of respiratory motor discharge have recently been described in neonates and are suggested to be a consequence of pharmacological blockade of multi- ple rhythm generators underlying respiratory rhythm (Mellen et al., 2003) and fractionated breaths and episodic breathing have been seen in in vitro neonatal rat brainstem preparations at lower temperatures (Mellen et al., 2002). Since hibernators in vivo exhibit episodic breathing as adults, both during hibernation at reduced temperature (body temperature $=5-20{ }^{\circ} \mathrm{C}$ ) (Zimmer and Milsom, 2000) and during severe hypothermia (body temperature $=5-10^{\circ} \mathrm{C}$ ) (Zimmer and Milsom, 2002), the production of episodic breathing at low temperatures may be a characteristic retained from birth in these species, just as cold tolerance appears to be. Hence, we predicted that hamster brainstem-spinal cord preparations would also produce episodic respiratory motor discharge from birth and that the expression of episodic breathing would not decrease with age.

\section{Methods}

\subsection{Animals and tissue preparation}

Animal care and experimental protocols were approved by the Animal Care Committee of the University of British Columbia acting under the guidelines set by the Canadian Council for Animal Care. Pregnant Syrian hamsters were purchased from Charles River Rodent Laboratories (Calgary, AB, Canada) and allowed to give birth naturally. In captivity, this species will only enter hibernation if environmental conditions are changed in an appropriate fashion. Our animals were housed at $21^{\circ} \mathrm{C}$ and constant photoperiod (12 h light: $12 \mathrm{~h}$ dark). Under these conditions they do not enter hibernation but breed throughout the year. Neonatal hamsters (age 0-6 days, body weights ranging from 3.1 to $9.0 \mathrm{~g}$ ) were deeply anesthetized with halothane (3-4\%), decerebrated, and the brainstem-spinal cords carefully dissected under constant superfusion $(5 \mathrm{ml} / \mathrm{min})$ with artificial cerebral spinal fluid (ACSF) composed of (in mM) 113.0 sodium chloride, 3.0 potassium chloride, 1.2 sodium phosphate, 1.5 calcium chloride, $1.0 \mathrm{mag}$ nesium chloride, 30.0 sodium bicarbonate and 30.0 dextrose, and equilibrated with $95 \%$ oxygen and 5\% carbon dioxide ( $\mathrm{pH} \sim 7.36$ at $21^{\circ} \mathrm{C}$ ). The brainstem was then cut either just rostral to the pons or at the pontomedullary junction, and the spinal cord was 
transected at the level of the seventh cervical root. The brainstem-spinal cord preparation was then pinned ventral side up in a recording dish and oxygenated ACSF was passed through a temperature controlled bath regulated with a Lauda cooler (Model RC6).

A glass pipette suction electrode was attached to the first cervical ventral root $(\mathrm{C} 1)$ to record respiratory-related motor output associated with inspiration and a thermistor was placed in the bath next to the brainstem to monitor bath temperature $\left(T_{\mathrm{b}}\right)$. Electrical signals recorded from suction electrodes were amplified, filtered and recorded (750 samples/s) on Windaq computer data acquisition software (DI200; DataQ Instruments, Akron, OH, USA).

\subsection{Experimental protocol}

Each brainstem-spinal cord preparation was dissected at room temperature $\left(19-25^{\circ} \mathrm{C}\right)$. Our standard protocol was to remove the pons initially and record respiratory-related motor activity from $\mathrm{C} 1$ for 20-30 min. The preparation was then warmed to $27^{\circ} \mathrm{C}$ and baseline activity was recorded for another 20-30 min before starting a cooling and warming protocol. In one series of experiments, however, the pons was left on initially while we recorded at room temperature for $20-30 \mathrm{~min}$. We then warmed the preparation and waited another $20-30 \mathrm{~min}$. The pons was then removed slowly $(n=6)$ by first slicing through the left side of the pons followed by $5 \mathrm{~min}$ of recording and then the entire pons was removed followed by another $5 \mathrm{~min}$ of recording. This was the only group of preparations that had the pons intact.

One set of brainstem-spinal cord preparations was exposed to a rapid, continuous cooling protocol ( $n$ $=18$ ) in which the bath temperature was cooled at the rate of the Lauda cooler $\left(\sim 0.75^{\circ} \mathrm{C} / \mathrm{min}\right)$ until fictive breathing stopped and then warmed back to $27^{\circ} \mathrm{C}$. A second set of brainstem-spinal cord preparations was exposed to a slower, stepwise cooling protocol ( $n$ $=11$ ) in which the bath temperature was maintained at a steady temperature of $27^{\circ} \mathrm{C}$ for $20 \mathrm{~min}$, the temperature was then reduced in $4{ }^{\circ} \mathrm{C}$ steps, each step lasting $20 \mathrm{~min}$, until fictive breathing stopped. The preparation was then warmed in the reverse sequence of steps. During cooling and warming, the $\mathrm{pH}$ of the ACSF was allowed to fluctuate naturally with changes in temperature ( $\mathrm{pH}$ range $\sim 7.42$ at $27^{\circ} \mathrm{C}$ to $\sim 7.30$ at $13^{\circ} \mathrm{C}$ ).

\subsection{Data analysis}

The data from 0 to 3 days old hamster brainstemspinal cord preparations were combined to form a young group ( $n=10$, rapid, continuous cooling; $n$ $=5$, slower, stepwise cooling) and 4-6 days old hamster brainstem-spinal cord preparations were combined to form an older group ( $n=8$, rapid, continuous cooling; $n=6$, slower, stepwise cooling) to evaluate age effects on the response to hypothermia. Raw nerve recordings were full wave rectified and integrated. Each integrated burst of activity was analyzed for its duration, peak amplitude and area, and the total burst frequency calculated. To examine the influence of the pons on respiratory discharge, data were averaged for 5 min each with the pons intact, after hemisection, and after full transection. During rapid, continuous cooling, each integrated respiratory-like motor burst was analyzed and averaged for each minute during the entire cooling and re-warming protocol. During the slower, stepwise cooling, at least 30 individual bursts at each temperature were analyzed except when the frequency became extremely slow, whence the complete 20-30 min of recorded trace was analyzed. Data were only analyzed at 27,23 and $19^{\circ} \mathrm{C}$ during the stepwise cooling protocol because the bursts became highly irregular at colder temperatures (see Section 3). Because of the variability in electrical neural recordings between preparations, the data collected during cooling and warming were normalized to starting values at $27^{\circ} \mathrm{C}$ for each preparation for burst amplitude and area.

\subsection{Statistical analysis}

To examine the effect of temperature on breathing frequency, burst duration, amplitude and area, a one-way, repeated measures analysis of variance (ANOVA) was performed followed by a Bonferroni post hoc test. All data are presented as means \pm S.E. and $P<0.05$ was considered significant.

\section{Results}

\subsection{Pontine influences on fictive breathing}

Respiratory-like motor discharge was completely absent while the pons remained intact in neonatal ham- 


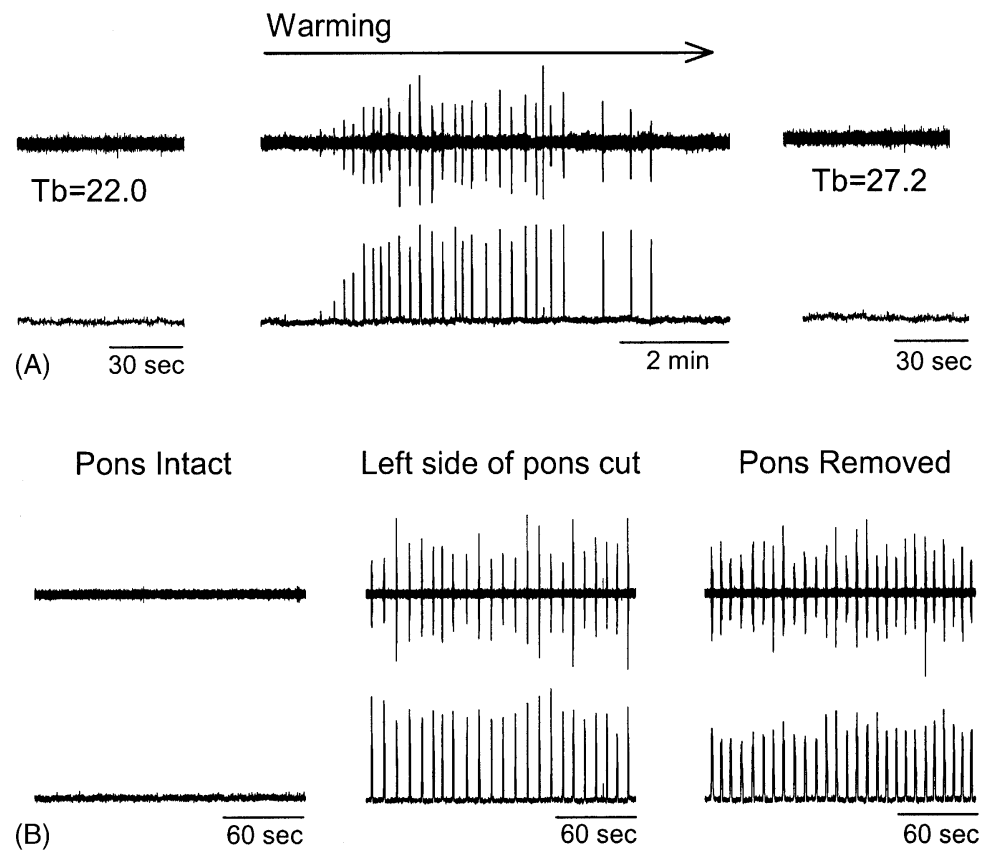

Fig. 1. Raw (top) and integrated (bottom) traces of respiratory-like neural discharge recorded from first cervical spinal nerves showing: (A) the effect of transiently warming the hamster brainstem-spinal cord preparation with the pons intact (4 days old) and (B) the effect of removing the pons at constant temperature $\left(T_{\mathrm{b}}=27^{\circ} \mathrm{C}\right)(2$ days old $)$.

ster brainstem-spinal cord preparations of all ages (0-6 days old) under steady state conditions. Warming preparations from 23 to $27^{\circ} \mathrm{C}$, however, did initiate respiratory-like activity transiently in some "pontine" preparations with this activity lasting from 2 to 20 bursts. This was only temporary, however, and once temperature began to stabilize at a new level, the fictive breathing stopped (Fig. 1A). Hemisection at the pontomedullary border subsequently resulted in the appearance of a stable fictive breathing rhythm (Fig. 1B) and when the entire pons was removed, the frequency of this rhythm increased significantly. This was due to a significant reduction in the expiratory pause between bursts (Figs. 1B and 2). Burst duration and amplitude remained relatively constant (Fig. 2).

\subsection{Effect of hypothermia on fictive breathing_rapid, continuous cooling}

At $27^{\circ} \mathrm{C}$, the frequency of respiratory-like motor discharge from young, hamster brainstem-spinal cord preparations was significantly faster than the frequency from older preparations. During fast, continu- ous cooling, the frequency decreased progressively in both younger and older preparations until respiratory arrest occurred. The older hamster brainstem-spinal cord preparations were more cold tolerant than the younger preparations; their respiratory-like activity did not stop until significantly colder temperatures were reached (Fig. 3). As frequency decreased during cooling in young brainstem-spinal cord preparations, the burst parameters remained relatively constant (Fig. 4). Although there was a small, significant increase in peak amplitude, this did not result in an increase in burst area. Older brainstem-spinal cord preparations, however, showed greater changes in burst variables during cooling; the duration of each burst of activity increased significantly and this resulted in a significant increase in burst area while peak amplitude remained unchanged (Fig. 4).

In a large percentage of young preparations (70\%), respiratory-like motor bursts became episodic during rapid, continuous cooling (Fig. 5). The remaining preparations exhibited either slow, regular bursts of activity $(10 \%)$ or an intermediate pattern $(20 \%)$ containing occasional clusters of bursts. In older 

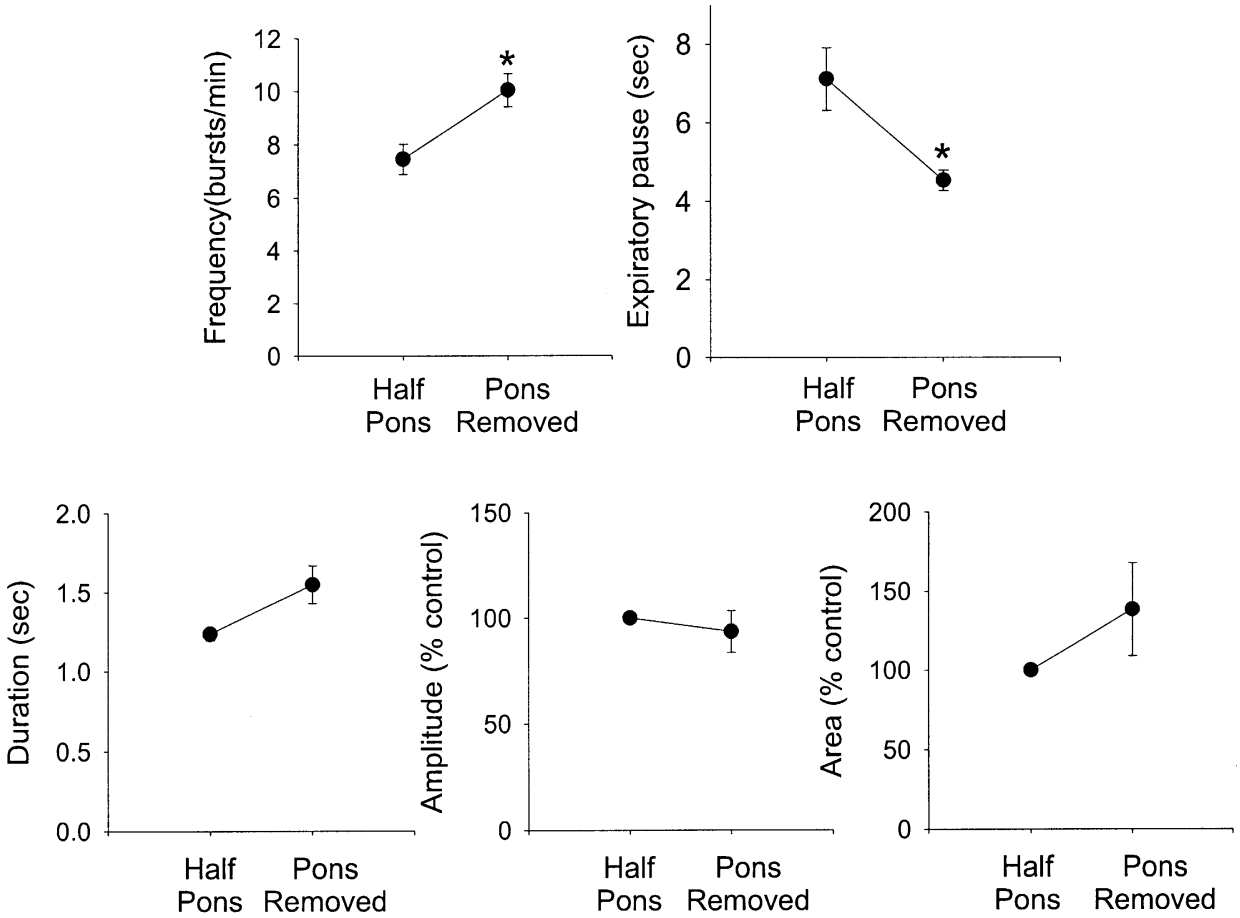

Fig. 2. The effect of removing the pons of the hamster brainstem-spinal cord preparation on the fictive breathing frequency, the expiratory pause, the inspiratory duration, the peak amplitude, and the integrated burst area $\left(T_{\mathrm{b}}=27^{\circ} \mathrm{C}\right)(n=6)$. An asterisk $(*)$ denotes a significant difference between preparations with half a pons or no pons. There was no respiratory motor output with the whole pons intact.

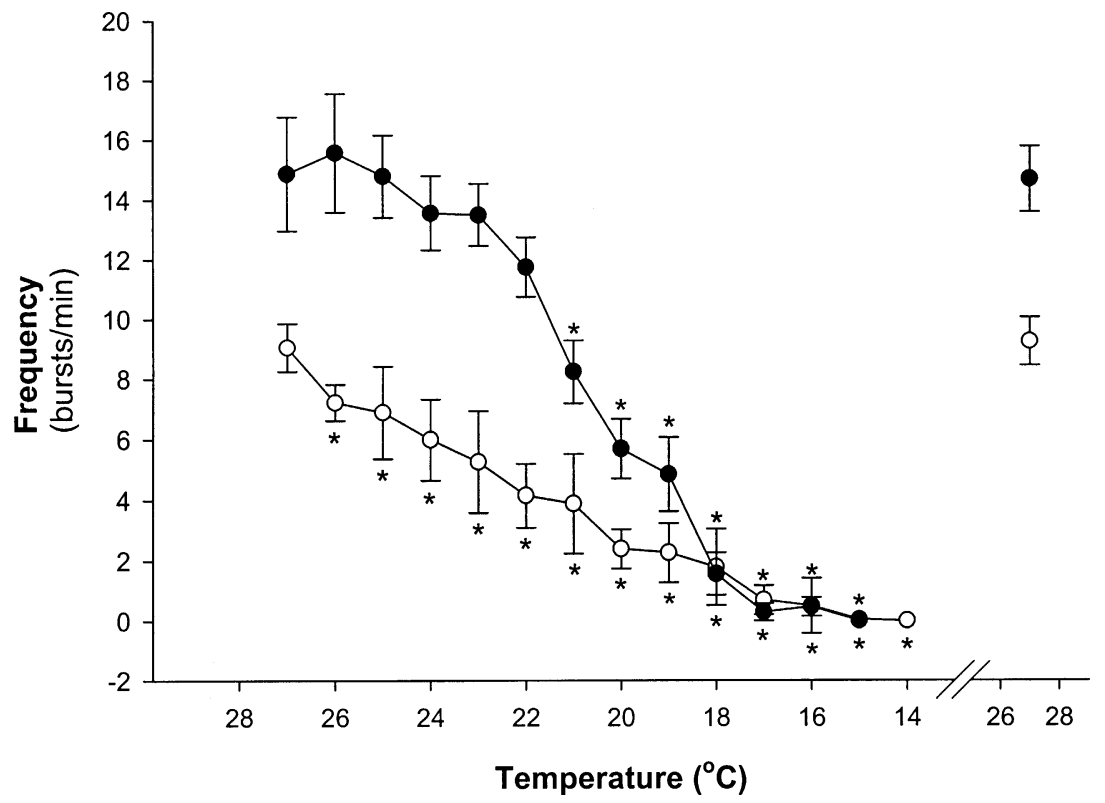

Fig. 3. The effect of cooling and warming the brainstem spinal cord on fictive breathing frequency. An asterisk $(*)$ denotes a significant difference from starting values. Filled circles $=$ preparations from young hamsters $(0-3$ days of age, $n=10)$; open circles $=$ preparations from older hamsters (4-6 days of age, $n=8)$. 

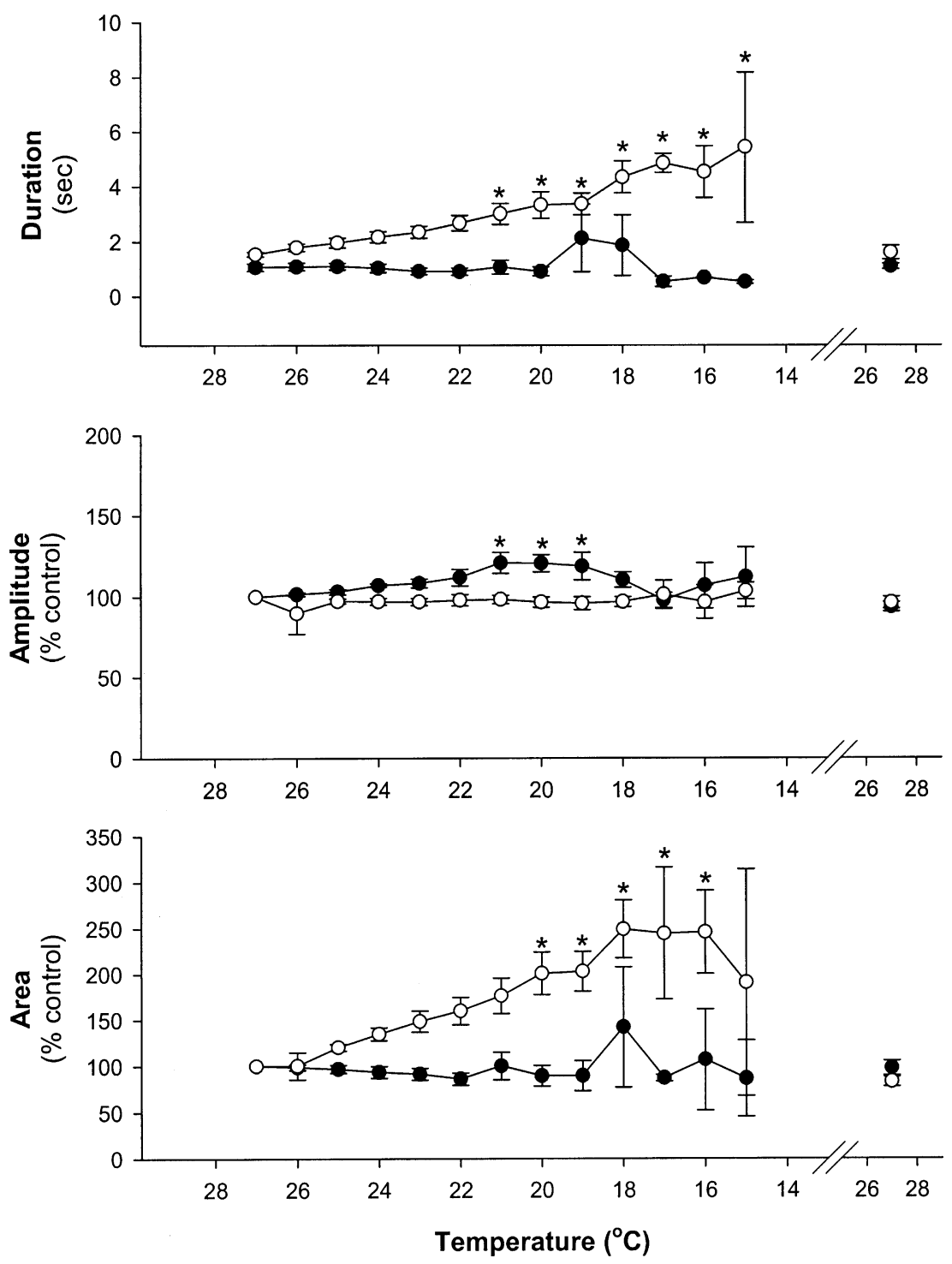

Fig. 4. The effect of rapid, continuous cooling on the burst duration, amplitude, and area of respiratory-like discharge in hamster brainstem-spinal cord preparations. An asterisk $(*)$ denotes a significant difference from starting values. Filled circles $=$ preparations from young hamsters ( $0-3$ days of age, $n=10)$; open circles = preparations from older hamsters (4-6 days of age, $n=8)$.

preparations, episodic patterns of activity were never observed and the majority of preparations exhibited slow, regular bursts of motor output (75\%). Occasionally, however, some bursts became clustered as in the intermediate pattern $(25 \%)$.

Finally, the burst profile of the respiratory-like motor discharge was primarily bell-shaped at $27^{\circ} \mathrm{C}$, although sometimes decrementing discharge profiles were observed (Fig. 6). The rapid onset, decrementing pattern which is so commonly observed in rat and mouse brainstem-spinal cord preparations was only observed after hemisection at the pontomedullary border and eventually bursts became more rounded as the entire pons was removed. During cooling, the burst shape tended to become more rapid in onset and decrementing in profile (see Fig. 5). 


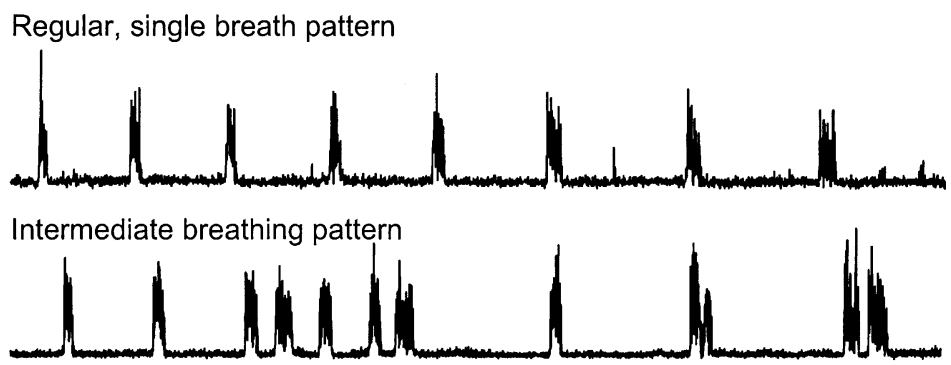

Episodic breathing pattern

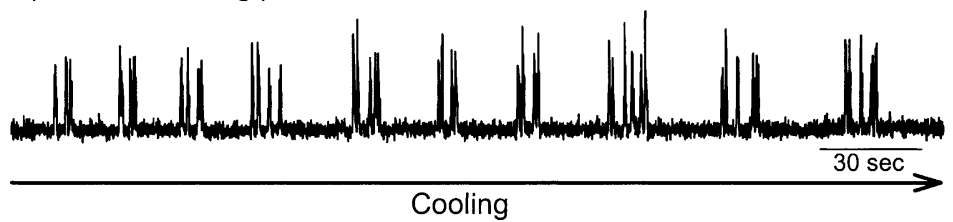

Fig. 5. Rapid, continuous cooling resulted in three different types of fictive breathing patterns in hamster brainstem-spinal cord preparations; regular, intermediate and episodic. The top two traces, from older neonates, and the bottom, episodic trace from a young neonate also show the difference in the burst duration and shape during cooling between the two different age groups.

\subsection{Effect of rapid, continuous rewarming on fictive breathing}

Rapidly and continuously rewarming hamster brainstem-spinal cord preparations following respiratory arrest restored the respiratory-like motor discharge. The manner in which breathing was restored varied, however (Fig. 7). In a few young brainstemspinal cord preparations fictive breathing started back slowly and then increased in amplitude and frequency before stabilizing again at $27^{\circ} \mathrm{C}$ (Fig. 7A). In the majority of preparations, however, young and old, a massive burst of neural activity (seizure or spasm-like discharge) occurred at some time during the warming phase. Sometimes the respiratory-like discharge was superimposed on top of this increased activity but most of the time the fictive breathing became masked by the increased background noise. This was followed either by the resumption of normal respiratory-like activity (Fig. 7B) (only observed in young prepara-

Bell shaped discharge
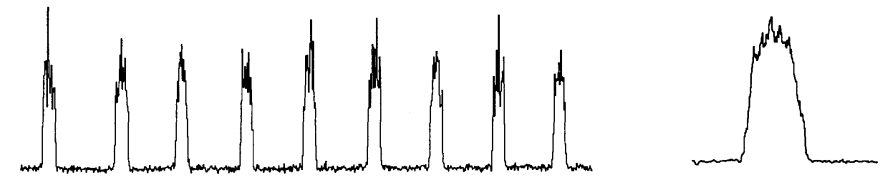

Decrementing shaped discharge
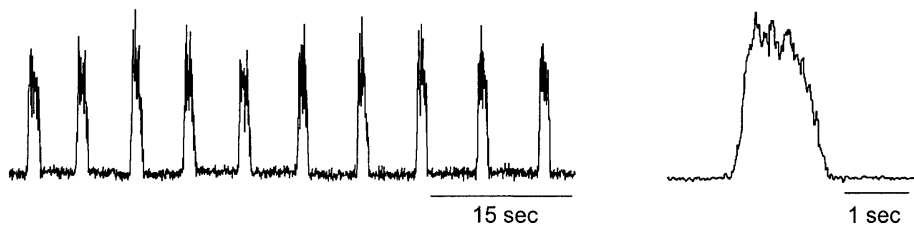

Fig. 6. Examples of the shapes of burst discharge typically observed in hamster brainstem-spinal cord preparations (both examples are from older preparations). The right hand trace is the average of 10 bursts of respiratory-like activity. 


\section{Warming}

(A)

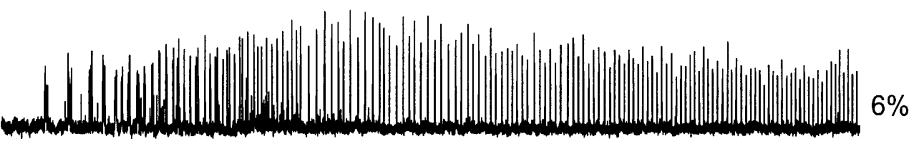

(B)

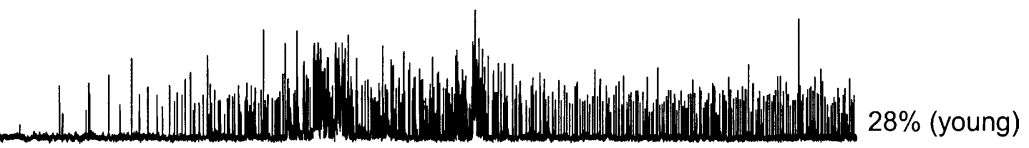

(C)

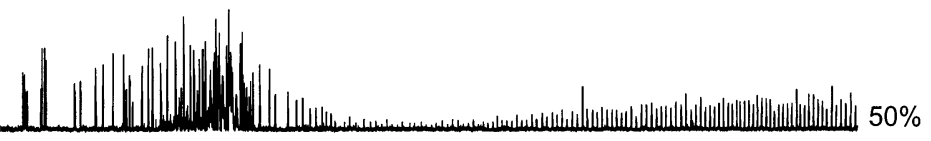

(D)

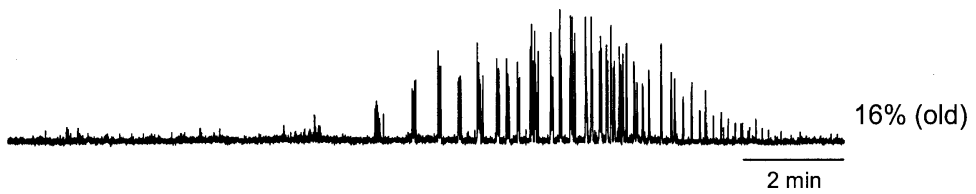

Fig. 7. Examples of the various fictive breathing patterns exhibited during rapid, continuous warming in hamster brainstem-spinal cord preparations. The percentages of preparations exhibiting each type of discharge are noted. See text for full descriptions.

tions), a pattern in which the burst amplitude was greatly reduced and then slowly returned to normal while frequency increased normally (Fig. 7C), or a pattern in which amplitude and frequency decreased until fictive breathing stopped and never resumed (Fig. 7D) (only observed in older preparations). Variability in the time of occurrence and length of the massive discharge between preparations made analysis and interpretation of the respiratory-like burst frequency, duration, peak amplitude and area difficult during the rewarming phase. Thus, fictive breathing was only analyzed once steady values were reached at $27^{\circ} \mathrm{C}$, except, of course, in those older preparations in which respiratory-like motor activity did not return $(16 \%)$.

\subsection{Effect of slower, stepwise cooling and rewarming on fictive breathing}

Slower, stepwise cooling and warming produced slightly different results than rapid, continuous cooling and rewarming. Fictive breathing frequency still decreased progressively as hamster brainstem-spinal cord preparations were cooled in $4{ }^{\circ} \mathrm{C}$ steps, and respiratory arrest occurred in all preparations. However, there were no significant changes in the duration, peak amplitude and area of the respiratory-like motor burst activity during the stages of cooling examined $\left(27,23\right.$ and $19^{\circ} \mathrm{C}$ ) regardless of age (Fig. 8). At the lowest step $\left(16^{\circ} \mathrm{C}\right)$, the burst activity became highly irregular; some bursts contained only a few spikes of activity, some contained large, long bursts of activity and some contained staccato like bursts of activity (data not shown). This irregular "bursting" type of activity was hard to quantify, especially since it was not clear whether it was distinctly respiratory in nature. It appeared, however, that the temperature at which all types of "bursting" activity stopped was not different than in the progressive cooling protocol. During stepwise rewarming, several preparations exhibited a small amount of the seizure-like neural activity that was commonly observed during rapid, continuous warming, however, once a stable temperature step was completed, the seizure activity disappeared and fictive breathing became regular. Finally, no clear episodic discharge patterns were observed during slower, stepwise temperature changes. Some clustering of breaths was seen immediately following step changes during cooling, but once temperature stabilized, the pattern of respiratory motor discharge became regular again (data not shown). 

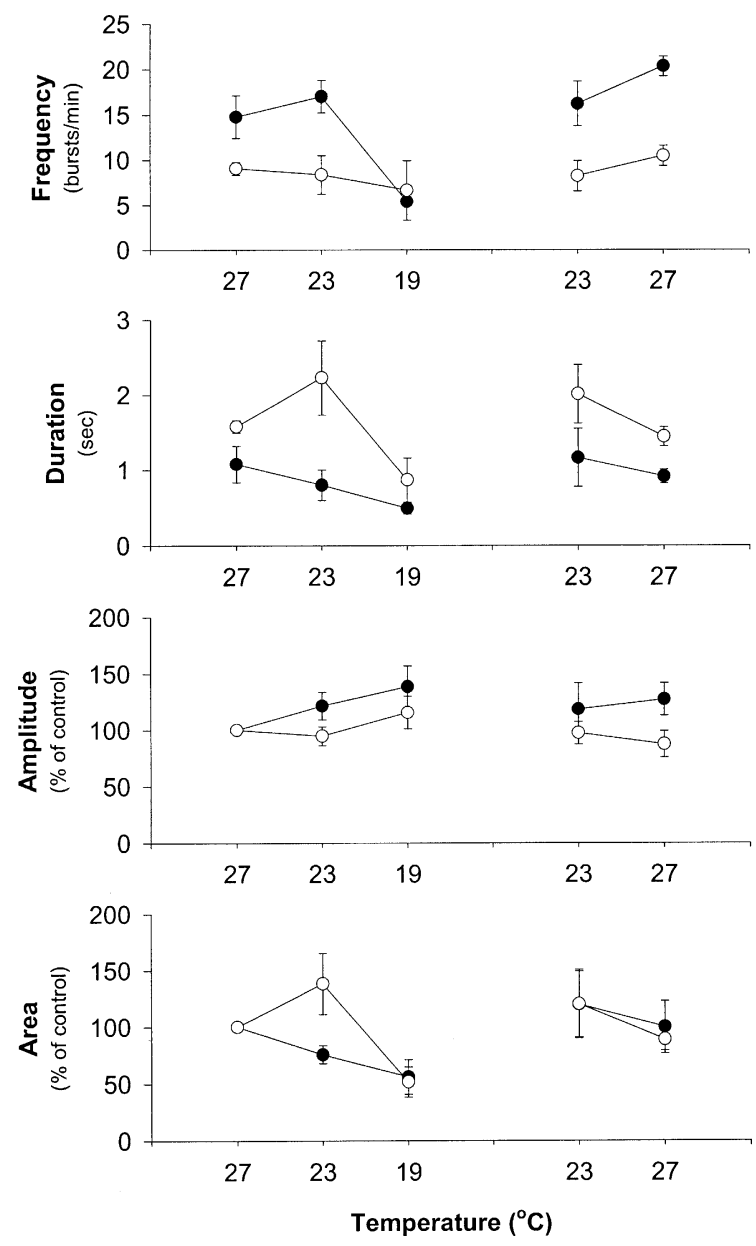

Fig. 8. The effect of slower, stepwise cooling and warming on the fictive breathing frequency, burst duration, burst amplitude and burst area of young ( $0-3$ days old, filled symbols, $n=5$ ) and old (4-6 days old, open symbols, $n=6$ ) hamster brainstem-spinal cord preparations.

\section{Discussion}

\subsection{Critique of methods}

It has been shown that the neonatal rat brainstemspinal cord preparation has a hypoxic core (Okada et al., 1993). Given the relatively small size of the hamster brainstem-spinal cord preparation, hypoxia may be less of a concern with this preparation, but oxygen concentrations were not measured in this study. Tissue $\left[\mathrm{CO}_{2}\right]$ and $\mathrm{pH}$ will also be progressively elevated with distance from the surface of the preparation
(Okada et al., 1993). None the less, it has been shown that in vitro brainstem spinal cord preparations retain a significant response to reducing ACSF $\mathrm{pH}$. Recently, Infante et al. (2003) showed that reducing $\mathrm{pH}$ from 7.4 to 7.3 at constant temperature $\left(24-25^{\circ} \mathrm{C}\right)$ in a neonatal mouse preparation caused a significant increase in respiratory-like frequency. This is the same range over which the $\mathrm{pH}$ of our ACSF changed during cooling. As a result, a decreasing $\mathrm{pH}$ may have increased respiratory drive as temperature fell and helped to sustain the respiratory rhythm at colder temperatures. The extent to which this may have varied with preparations from animals of different ages is not clear at this time.

\subsection{Pontine influences on fictive respiratory rhythm}

Inputs from the pons (the medial parabrachial and Kölliker-Fuse nuclei, collectively referred to as the parabrachial complex $(\mathrm{PbC})$ and the A5 noradrenergic nucleus) are well known to influence breathing in vivo (see Bianchi et al., 1995 for review; Jodkowski et al., 1997) and in vitro (see Ballanyi et al., 1999 for review). In rat brainstem-spinal cord preparations, inputs arising from the A5 noradrenergic nucleus act to reduce fictive breathing frequency (Hilaire et al., 1989). Similar to mouse in vitro preparations (Hilaire et al., 1997), respiratory activity was completely inhibited by inputs arising from the pons in hamster brainstem-spinal cord preparations. In the mouse, the inhibition was attributed to inputs arising from the A5 nucleus, although, this was never directly demonstrated. Inhibitory inputs also arise from the $\mathrm{PbC}$ and even inputs from pontine reticular neurons influence breathing (see Bianchi et al., 1995 for review). Thus, more directed research is needed to assess whether the total inhibition arising from the pons in these latter two species is due to a greater inhibition arising from the A5 nucleus, greater inhibition arising from other inputs, or simply less motor output arising from the respiratory rhythm generator such that similar levels of inhibitory pontine influences override the rhythm.

Interestingly however, during transitional re-warming, hamster brainstem-spinal cords transiently initiated a respiratory-like motor rhythm when the pons remained intact, but its expression was only temporary and stopped as the temperature stabilized at any new value (see Fig. 1). The mechanism by which a transient increase in temperature could override inhi- 
bition of respiratory-like motor output and initiate fictive breathing is not clear. Also, when the pons was removed a large burst of neural activity, sometimes with a respiratory-like rhythm superimposed on it, was observed during the rapid, continuous re-warming phase suggesting that the brainstem neural network as a whole may be hyper-excitable during rapid and transient warming. One possible explanation of these two phenomena is that the resting membranes of motor and other neurons are depolarized at lower temperatures. This would bring the membrane closer to threshold and would require less synaptic input to produce a motor response. When the brainstem-spinal cord preparations are subsequently warmed, increases in synaptic activity may precede membrane repolarization, making neurons relatively more excitable during the rapid, continuous warming phase. Indeed, in hippocampal slice preparations, pyramidal neurons depolarize at lower temperatures and repolarize during re-warming (Aihara et al., 2001).

Regardless of the mechanism, a more excitable respiratory system could be advantageous to a hibernator both during hibernation and during arousal from hibernation. During deep hibernation, when energy conservation is at a maximum, a system that required less synaptic input to produce resting breathing and respiratory responses would be most beneficial. Even during arousal a "feed-forward neural mechanism" could contribute to the tight correlation between the rapid increases in ventilation and metabolism that are observed. There is growing evidence which suggests that factors other than metabolism may be involved in setting the resting level of ventilation during deep hibernation (McArthur and Milsom, 1991; Zimmer and Milsom, 2000). Whether this increased neural excitability is unique to hibernators and aids in hibernation and arousal is not known.

\subsection{Effect of hypothermia on respiratory-like motor activity}

Two protocols were used to induce hypothermia in hamster brainstem-spinal cord preparations; rapid, continuous cooling and slower, stepwise cooling. Fictive breathing arrested during hypothermia and spontaneously recovered during rewarming during both protocols. During rapid, continuous cooling, motor burst duration and area increased significantly in older preparations while only the burst amplitude increased in younger preparations. Similar results have been reported for neonatal rat in vitro preparations (Mellen et al., 2002). However, during the slower, stepwise cooling protocol there were no significant changes in any of the respiratory-like burst variables. The significance of different changes in burst variables as a function of the rate of cooling is unclear. In vivo, during entrance into hibernation, body temperature falls quite slowly taking between 12 and $20 \mathrm{~h}$ before minimum body temperatures are reached (Wang and Hudson, 1971). Ventilation slows through changes in frequency and pattern alone while tidal volume remains constant. Even during hypothermia, the tidal volume of neonatal rats in vivo stays relatively constant during cooling and only increases just before arrest at low temperatures (Tattersall and Milsom, 2003). This might suggest that the data from the slower, stepwise cooling in the present study more closely mimics cooling during entrance into hibernation. However, the burst activity became highly irregular at the lowest temperatures during stepwise cooling, but stayed regular during the progressive cooling more closely mimicking physiological changes. Since body temperature falls progressively, but very slowly during entrance into hibernation, a very slow progressive fall in temperature may have produced more consistent results. Clearly more work is needed to resolve why and how different patterns of cooling produce different changes in respiratory-like burst activity.

The temperature at which fictive breathing arrested was lower in older hamster brainstem-spinal cord preparations (4-6 days old) than in younger preparations (0-3 days old) during progressive cooling despite having a lower starting frequency. This also appeared to be the case in the stepwise cooling protocol but since it was hard to discern the "respiratory" bursts at the lowest temperature with this protocol, this is still open for debate. This suggests that as the neurons of the respiratory network mature, a more stable network arises that is more cold tolerant. It is not clear whether this is unique to the hibernating species or present in all developing species. Mellen et al. (2002) showed that the temperature at which fictive breathing stopped in young rat in vitro brainstem spinal cord preparations (0-3 days old) was similar to this study (Fig. 9) and results obtained in vivo (rat, Tattersall and Milsom, 2003) suggested that adapta- 


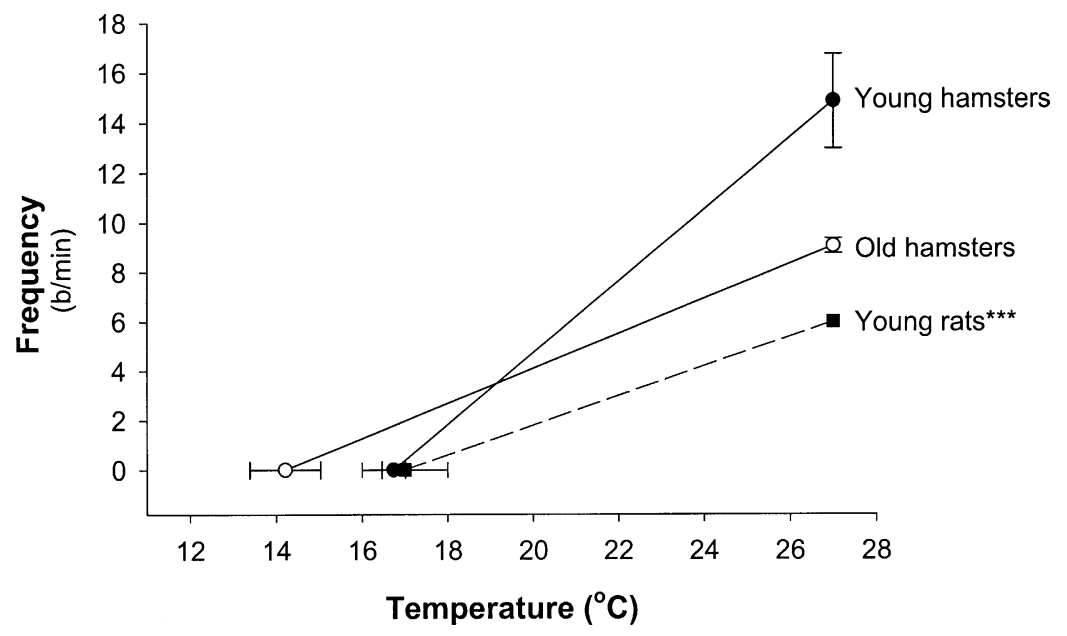

Fig. 9. Comparison of the starting breathing frequencies and the temperatures at which breathing arrested in young (0-3 days old) and old (4-6 days old) hamster brainstem-spinal cord preparations compared to young rat brainstem-spinal cord preparations (0-3 days old) (rat data taken from Mellen et al., 2002).

tions to the cold that occur in hibernating species are most notable in adults where the difference in the temperature at which breathing stops is much greater for the rat $\left(17-27^{\circ} \mathrm{C}\right)$ than for the hamster $\left(<5^{\circ} \mathrm{C}\right)$. Since the data obtained from both young hamster and rat in vitro preparations show similar results, this raises the intriguing possibility that adult hibernators are able to function in the cold because they retain neonatal characteristics.

\subsection{The effect of re-warming and auto-resuscitation from hypothermic arrest}

Rewarming hamster brainstem-spinal cord preparations resulted in spontaneous recovery of respiratorylike motor discharge in all preparations. The mechanistic basis of this ability to restart a fictive breathing rhythm is not known. As stated earlier, over $90 \%$ of brainstem-spinal cord preparations showed a massive spontaneous burst of generalized neural activity during the rewarming phase. While all preparations showed some sort of breathing rhythm during the initial stages of rewarming, not all continued to exhibit motor discharge throughout the entire warming phase; some older preparations stopped producing respiratory motor discharge after warming. This may reflect further maturation of the system since all adults, hibernators and non-hibernators lose the ability to spontaneously auto-resuscitate (Adolph, 1951).

\subsection{The effect of hypothermia on the generation of episodic patterns of fictive breathing}

Hamster brainstem-spinal cord preparations exhibited episodic respiratory-like motor discharge patterns during rapid, continuous cooling and re-warming but not during slower, stepwise cooling and re-warming. Episodic patterns were also observed occasionally during rapid, continuous cooling and re-warming of neonatal rat brainstem-spinal cord preparations (Mellen et al., 2002). It is not clear why the hamster preparations show a difference in the expression of episodic patterns based on the mode of cooling, especially since hibernators in vivo breathe episodically both during slow, progressive cooling during entrance into hibernation and during steady state changes in temperature during hibernation itself.

During rapid, continuous cooling and re-warming, episodic fictive breathing was observed in a greater percentage of young hamster brainstem-spinal cord preparations compared to older hamster preparations; $70 \%$ of young hamster preparations expressed episodic patterns during cooling, whereas none of the older preparations did. Breathing patterns are relatively unstable and occasional apneas are not uncommon in neonatal mammals (Hilaire and Duron, 1999). In adult hibernators pontine inputs have been speculated to play an important role in the production of episodic breathing (Milsom et al., 1997). The pons had 
to be removed in all hamster brainstem-spinal cord preparations to produce a fictive breathing rhythm suggesting that pontine inputs are strongly inhibitory under these conditions. It will be interesting to determine whether the mechanisms underlying episodic pattern formation in neonatal brainstem-spinal cord preparations are the same mechanisms underlying episodic breathing observed in adult hibernators.

\section{Conclusions}

Pontine inputs in hamster brainstem-spinal cord preparations completely inhibited respiratory-like motor rhythm arising from the medulla. Increasing the temperature, however, caused a temporary restoration of a fictive breathing rhythm. Similarly, increasing the temperature after cooling caused a massive discharge of neural activity in preparations with the pons removed. The mechanism behind this heightened respiratory sensitivity to increases in temperature is not known but may be advantageous to a hibernating mammal in which increases in body temperature may facilitate an increase in ventilation to support the massive increase in metabolism which occurs during arousal from hibernation.

Cooling hamster brainstem-spinal cord preparations reduced fictive breathing frequency, produced episodic patterns of motor discharge and was followed by arrest. Episodic patterns were only observed in young preparations during rapid, continuous cooling and not in older preparations, and not at all during slower stepwise cooling. It is not clear whether the episodic patterns observed in neonatal brainstem-spinal cord preparations are produced by the same mechanisms that produce episodic breathing in adults during hibernation. Finally, older hamster brainstem-spinal cord preparations were more cold tolerant suggesting that maturation of the respiratory neural network increases their cold tolerance. This can only occur up to a point, however, since cold tolerance ultimately decreases with age in mammals in vivo.

\section{Acknowledgements}

This work was funded by the NSERC of Canada.

\section{References}

Adolph, E.F., 1948a. Tolerance to cold and anoxia in infant rats. Am. J. Physiol. 155, 366-377.

Adolph, E.F., 1948b. Lethal limits of cold immersion in adult rats. Am. J. Physiol. 155, 378-387.

Adolph, E.F., 1951. Responses to hypothermia in several species of infant mammal. Am. J. Physiol. 166, 75-91.

Aihara, H., Okada, Y., Tamaki, N., 2001. The effects of cooling and rewarming on the neuronal activity of pyramidal neurons in guinea pig hippocampal slices. Brain Res. 893, 36-45.

Ballanyi, K., Onimaru, H., Homma, I., 1999. Respiratory network function in the isolated brainstem-spinal cord of newborn rats. Prog. Neurobiol. 59, 583-634.

Bianchi, A.L., Denavit-Saubie, M., Champagnat, J., 1995. Central control of breathing in mammals: neuronal circuitry, membrane properties, and neurotransmitters. Physiol Rev. 75, 1-45.

Hilaire, G., Duron, B., 1999. Maturation of the mammalian respiratory system. Physiol. Rev. 79 (2), 325-360.

Hilaire, G., Monteau, R., Errchidi, S., 1989. Possible modulation of the medullary respiratory rhythm generator by the noradrenergic A5 area: an in vitro study in the newborn rat. Brain Res. 485, 325-332.

Hilaire, G., Bou, C., Monteau, R., 1997. Rostral ventrolateral medulla and respiratory rhythmogenesis in mice. Neurosci. Lett. 224, 13-16.

Infante, C.D., von Bernhardi, R., Rovegno, M., Llona, I., Eugenín, J.L., 2003. Respiratory responses to $\mathrm{pH}$ in the absence of pontine and dorsal medullary areas in the newborn mouse in vitro. Brain Res. 984, 198-205.

Jodkowski, J.S., Coles, S.K., Dick, T.E., 1997. Prolongation in expiration evoked from ventrolateral pons of adult rats. J. Appl. Physiol. 82 (2), 377-381.

Lyman, C.P., 1982. Entering hibernation. In: Lyman, C.P., Willis, J.S., Malan, A., Wang, L.C.H. (Eds), Hibernation and Torpor in Mammals and Birds. Academic Press, New York, pp. 37-54.

McArthur, M.D., Milsom, W.K., 1991. Changes in ventilation and respiratory sensitivity associated with hibernation Columbian (Spermophilus columbianus) and golden-mantled (Spermophilus lateralis) during the summer and winter. Physiol. Zool. 64 (4), 921-939.

Mellen, N.M., Milsom, W.K., Feldman, J.L., 2002. Hypothermia and recovery from respiratory arrest in a neonatal rat in vitro brain stem preparation. Am. J. Physiol. 282, R484-491.

Mellen, N.M., Janczewski, W.A., Bocchiaro, C.M., Feldman, J.L., 2003. Opioid-induced quantal slowing reveals dual networks for respiratory rhythm generation. Neuron. 37, 821-826.

Milsom, W.K., Harris, M.B., Reid, S.G., 1997. Do descending influences alternate to produce episodic breathing? Respir. Physiol. 110 (2-3), 307-317.

Okada, Y., Muckenhoff, K., Holtermann, G., Acker, H., Scheid, P., 1993. Depth profiles of $\mathrm{pH}$ and PO2 in the isolated brain stem-spinal cord of the neonatal rat. Respir. Physiol. 93, 315326.

Tattersall, G., Milsom, W.K., 2003. Hypothermia-induced respiratory arrest and recovery in neonatal rats. Respir. Physiol. Neurobiol. 137 (1), 29-40. 
Wang, L.H.C., Hudson, J.W., 1971. Temperature regulation in normothermic and hibernating eastern chipmunk, Tamias striatus. Comp. Biochem. Physiol. A.: Mol. Integr. Physiol. 38, 59-90.

Zimmer, M.B., Milsom, W.K., 2000. Effects of changing ambient temperature on metabolic, heart, and ventilation rates during steady state hibernation in golden-mantled ground squirrels
(Spermophilus lateralis). Physiol. Biochem. Zool. 74 (5), 714 723.

Zimmer, M.B., Milsom, W.K., 2002. Ventilatory pattern and chemosensitivity in unanesthetized, hypothermic ground squirrels (Spermophilus lateralis). Respir. Physiol. Neurobiol. 133, 49-63. 